\title{
Les représentations des compétences professionnelles: une première entrée en dialogue avec des enseignants franco-ontariens
}

\author{
Ann-Louise Davidson \\ Université d'Ottawa
}

\section{Résumé}

De nombreux écrits traitant des changements en cours dans le monde de l'éducation occidental ont permis de repérer les diktats de mode en ce qui a trait à l'amélioration de la qualité de l'enseignement. La province d'Ontario, influencée par ces tendances autant américaines qu'européennes, possède une histoire riche de restructurations politiques et sociales qui ont guidé la réforme éducative actuellement en oeuvre. Dans ce contexte, la présente étude s'est attachée à analyser l'écart entre les représentations des compétences professionnelles que des enseignantes et enseignants se sont construites à partir de leur expérience, et les définitions de compétences incluses dans les référentiels de Perrenoud $(1998,2002)$ et de Gauthier (Ministère de l'Éducation du Québec, 2002), ces référentiels possédant un lien étroit avec les compétences professionnelles en enseignement jugées essentielles par l'Ordre des enseignantes et des enseignants de l'Ontario. Menée à l'été 2002, la présente étude a constitué une entrée en dialogue avec des enseignants faisant partie de la première cohorte à entreprendre le Programme de perfectionnement professionnel imposé par l'Ordre. Les écarts retrouvés conduisent à s'interroger sur les facteurs de résistances au changement institutionnel tels que la tradition et l'inertie, mais aussi sur le rôle possible des contradictions entre discours politiques et discours professionnels entourant la réforme ontarienne (Routhier Boudreau, 2002, 2003).

\section{Summary}

Literature on current changes in the educational systems throughout the western world has contributed to the identification of the fashion diktats aimed at improving the quality of teaching. The province of Ontario, strongly influenced by these trends, possesses a rich history of political and social restructuring which have influenced the educational reform presently being implemented. In this context, the teachers' representations of their own professional competencies were analysed in parallel to Perrenoud's $(1998,2002)$ and Gauthier's (Ministère de l'Éducation du Québec, 2002) typologies of competencies in the teaching profession. The competencies identified by these authors have been closely related to the ones deemed essential by the Ontario College of Teachers. The present study, conducted during the summer of 2002, initiated a dialogue with the first cohort of teachers who were obligated to participate in the Professional Improvement Program. The discrepancies between the teachers' representations and the competencies put forward by the researchers raise questions about factors contributing to resistance to institutional change such as inertia and tradition, and also raise questions concerning the role of contradictions in political and professional discourses on educational reform in Ontario (Routhier Boudreau, 2002, 2003). 


\section{Introduction}

Au cours des quatre dernières décennies, les pays occidentaux ont connu de multiples réformes éducationnelles (UNESCO, 2001). Parmi celles-ci, la réforme ontarienne, en œuvre depuis 1997, a fait vivre aux enseignantes et aux enseignants à qui on a imposé la professionnalisation, une expérience plurivoque. D'une part, les discours politiques dans l'esprit de la Nouvelle Gestion Publique (NGP) visant l'accroissement de la qualité de l'éducation dans le but de former de meilleurs travailleurs pour le monde de demain, ont incité le Ministère de l'éducation ontarien à mettre en oeuvre une réforme rapide, pour obtenir des résultats instantanés (St-Germain, 2001a). D'autre part, selon les recherches actuelles en éducation, on constate que le changement significatif prend du temps, ce qui incite à adopter des méthodes différenciées qui permettent le développement de nouvelles compétences dans l'espace-temps (Perrenoud, 1993, 1998, 2002). Cet écart entre discours politique réformiste et recherche en éducation nous amène à entamer un dialogue avec les enseignants afin de mieux comprendre les représentations qu'ils se font de leurs compétences professionnelles.

Ce texte s'attache d'abord à présenter le contexte entourant la professionnalisation de l'enseignement en Ontario par rapport aux tendances mondiales et canadiennes, et à décrire le Programme de perfectionnement professionnel imposé par l'Ordre des enseignantes et des enseignants de l'Ontario. La question de recherche qui en découle concerne le changement dans les représentations des compétences professionnelles chez les enseignants engagés dans ce programme. La méthodologie utilisée dans cette étude est ensuite esquissée, puis les résultats sont analysés. La dernière section offre des pistes de réflexion sur les sources possibles de résistance au changement et sur le besoin d'une approche plus valorisante de la professionnalisation de l'enseignement.

\section{Contexte entourant la professionnalisation de l'enseignement en Ontario}

Dans les programmes d'études découlant des réformes des systèmes d'éducation des pays occidentaux (UNESCO, 2001), on observe des changements qui sont tributaires du contexte sociétal actuel marqué par la mondialisation de l'économie et par l'avancement des technologies de l'information et des communications. Cependant, tel que l'énonce Gohier (2002) dans un liminaire sur les finalités de l'éducation, les fondements des réformes qui devraient apparaître en amont de tout changement important dans les orientations d'un système éducatif, sont souvent formulés en aval, servant à rebours à des impératifs économiques, politiques, bureaucratiques, selon les diktats de la mode.

À cet égard, et dans les réformes mises de l'avant par les gouvernements des pays industrialisés, on observe un changement de direction quant aux pratiques éducationnelles. L'enseignement par compétences, les 
approches pédagogiques par projet et par problème, les évaluations formative et standardisée, sont des termes qui constituent un langage commun dans l'écriture des programmes d'études. De fait, en parcourant les sites Web des ministères de l'éducation de différents pays ou provinces dont, entre autres, l'Australie (Department of Education, Science and Training, 2004), la Grande Bretagne (Department of Education and Skills, 2004), la France (Ministère de l'éducation nationale, de l'enseignement supérieur et de la recherche, 2002) et le Québec (Ministère de l'éducation du Québec, 2004), on remarque que tous adoptent ce même langage. Ce langage veut répondre aux pressions croissantes du public ainsi qu'aux changements accélérés de l'ère des technologies. L'amélioration de la qualité de l'éducation exige des compétences pédagogiques plus appropriées pour les enseignants qui oeuvrent dans un système en changement tout en répondant aux attentes sociétales de réussite scolaire (Develay, 2001; St-Jarre et Dupuy-Walker, 2001).

\section{La perspective canadienne}

Dans le contexte canadien, afin de maximiser le développement des compétences chez le personnel enseignant et d'accroître la qualité de l'éducation, la création d'ordres professionnels est devenue une tendance marquée, du moins en Ontario et en Colombie-Britannique. En créant un ordre des enseignants, ces provinces reconnaissent automatiquement à ces derniers le statut de professionnels. Cette façon d'attribuer un tel statut ne fait pourtant pas l'unanimité partout au pays. Récemment, le Québec a refusé de créer un organisme d'autoréglementation pour le corps enseignant, sous prétexte que la profession est déjà bien encadrée par la loi sur l'éducation et les syndicats professionnels (Office des professions du Québec, 2002). Ce refus de créer un ordre professionnel pour les enseignants n'empêche cependant pas ceux-ci d'accéder au statut professionnel, puisque ce statut s'inscrit dans un contexte législatif. De fait, quelle que soit leur approche en matière de professionnalisation de l'enseignement, les provinces trouvent appui auprès des chercheurs, en accord avec les demandes accrues du milieu éducatif. Les recherches menées depuis une vingtaine d'années en éducation témoignent en effet de l'importance de professionnaliser l'enseignement, au même titre que les autres professions libérales (Perrenoud, 1998; Tardif et Gauthier, 1999).

La réponse du gouvernement Harris (élu le 9 juin 1995) à ce courant de professionnalisation a été de créer, en 1996, l'Ordre des enseignantes et des enseignantes de l'Ontario, dont certaines des fonctions étaient d'élaborer et maintenir les normes d'admissibilité à la profession, d'établir et faire respecter les normes professionnelles et les normes déontologiques applicables à ses membres, d'agréer les programmes de formation professionnelle et continue des enseignants offerts par les établissements d'enseignement postsecondaire, et d'assurer la formation continue de ses membres, notamment par un programme de perfectionnement professionnel obligatoire pour conserver le certificat de compétence en enseignement et demeurer membre de l'Ordre. Ce dernier programme a été particulièrement controversé. Par exemple, du côté anglophone, l'Ontario Secondary School Teacher Federation (OSSTF) déclare que ses membres sont désillusionnés face aux initiatives de l'Ordre, parce que le gouvernement s'est servi de cet organisme pour contrôler et punir les enseignants (OSSTF, 2004). À titre de porte-parole pour ses membres, I'Association des enseignants franco-ontariens (AEFO, 2003) soutient que la recertification (1) est inutile; (2) coûte cher; (3) porte atteinte au professionnalisme; (4) répond à des impératifs politiques plutôt que pédagogiques et (5) fait abstraction des problèmes importants. 


\section{Le Programme de perfectionnement professionnel imposé par l'Ordre des enseignantes et des enseignants de l'Ontario}

En mai 2000, l'Ordre des enseignantes et des enseignants de l'Ontario et le Ministère de l'éducation ont publié le Programme ontarien d'évaluation du personnel enseignant dont le but était de maintenir l'excellence en éducation. Ce programme décrivait les mesures à prendre afin que tout le personnel enseignant maintienne ses connaissances, ses compétences et ses habiletés à jour, afin de s'acquitter de ses responsabilités professionnelles (Ministère de l'éducation de l'Ontario, 2002). Ce programme comprenait des mesures pour l'évaluation du rendement, pour l'insertion des nouveaux membres dans la profession et pour le développement professionnel des enseignants en exercice.

Les mesures pour les enseignants en exercice incluaient une exigence de recertification à intervalle régulier. Pour conserver leur certificat de compétence, les enseignants devaient, tous les 5 ans, réussir quatorze cours, dont sept obligatoires et sept au choix (Gouvernement de l'Ontario, 1996). En plus de ces quatorze cours, les enseignants devaient subir des tests écrits et des évaluations connexes, et tenir un portfolio professionnel dont les composantes étaient déterminées par l'Ordre des enseignantes et des enseignants de l'Ontario (Ministère de l'Éducation de l'Ontario, 2004).

\section{Réactions des enseignants}

En parcourant les sites Web des associations professionnelles telles que l'Association des enseignants franco-ontariens (AEFO), l'Ontario Secondary School Teacher Federation (OSSTF), l'Ontario Teacher Federation (OTF), I'Elementary Teacher Federation of Ontario (OTFO), et l'Ontario English Catholic Teachers Association (OECTA), on constate l'univocité des discours en ce qui a trait au Programme de perfectionnement professionnel. Ces associations incitent leurs membres à ne pas participer au programme obligatoire mis de l'avant par l'Ordre des enseignantes et des enseignants de l'Ontario, à cause de son caractère trop formel et réglementé. À cet effet, ces associations ont mis à la disposition de leurs membres des modèles de lettres demandant aux organismes dispensant les cours de perfectionnement professionnel de ne pas envoyer d'information à leur sujet à l'Ordre des enseignantes et des enseignants de l'Ontario. Ces associations ont aussi élaboré des modèles de lettres destinées à la Ministre de l'éducation, Elizabeth Witmer, pour expliquer leur refus de participer au Programme de perfectionnement professionnel. Ces lettres expliquent que les enseignants croient au bien-fondé du perfectionnement professionnel, qu'ils cherchent toujours à améliorer leur enseignement, mais qu'ils veulent décider eux-mêmes des cours à suivre, étant les mieux placés pour décider de leurs besoins de formation. Somme toute, le Programme de perfectionnement professionnel a été vu comme une ingérence directe et « déprofessionnalisante » dans le travail des enseignants.

L'approche de la professionnalisation de l'enseignement du gouvernement Harris est d'ailleurs contraire aux principes de professionnalisation habituellement reconnus. En effet, « partout en Amérique du Nord, les professions sont considérées comme une avant-garde dans l'organisation du travail; elles constituent des groupes de travailleurs qui gèrent, de manière autonome et responsable, leurs activités et leurs règles de fonctionnement » (Tardif, 2004, p. 6). Avec le Programme de perfectionnement professionnel, l'Ordre des enseignantes et des enseignants de l'Ontario dirige un regard accusateur vers les enseignants et leur attribue les pauvres résultats scolaires des élèves ontariens (OSSTF, 2004), au lieu de faire reconnaître les enseignants publiquement comme des professionnels responsables et 
capables d'autorégulation de leur pratique. Le gouvernement McQuinty (élu le 2 octobre 2003) reconnaît d'ailleurs ce problème. Le 13 mai 2004, le ministre de l'éducation Gerard Kennedy déclare publiquement : « Nous voulons assurer l'excellence en enseignement à toutes nos écoles. La différence clé entre le gouvernement McGuinty et celui qui l'a précédé tient de ce que nous croyons que les enseignantes et enseignants le souhaitent également » (Gouvernement de l'Ontario, 2004). On peut donc s'interroger sur le caractère professionnalisant de la création d'un ordre professionnel lorsque celui-ci est régi par la haute administration d'un gouvernement interventionniste qui adopte une attitude de reproche envers les enseignants, même si le Ministère de l'éducation ontarien a automatiquement conféré le statut de professionnel aux enseignants, en passant la Loi de 1996 sur l'Ordre des enseignantes et des enseignants de l'Ontario.

\section{Contexte minoritaire franco-ontarien}

$\mathrm{Au}$ sein de ce tumulte politique, les enseignants franco-ontariens doivent conjuguer avec les exigences croissantes de leur profession et celles de leur travail d'enseignants en contexte linguistique minoritaire. Dans le contexte franco-ontarien actuel, les enseignants sont interpellés par les mêmes exigences de professionnalisation que la majorité anglophone. D'ailleurs, Gérin-Lajoie (2001) souligne que l'histoire des communautés francophones en milieu minoritaire a été marquée, entre autres, par l'obligation de vivre sous l'organisation politique et sociale de ceux qui représentent la plus grande majorité de la population. Sous l'emprise de l'Ordre des enseignantes et des enseignants de l'Ontario, les enseignants franco-ontariens n'ont donc d'autre choix que de se conformer aux exigences de professionnalisation, même si celles-ci sont controversées. Cette situation n'est pas anormale parce que, pour cette population, la survie linguistique et culturelle a toujours été une question de lutte contre les politiques anglosaxonnes dominantes, de prise de parole, voire de rébellion contre les diktats de la mode.

\section{Le but de la présente recherche}

Tenant compte de cette situation, il semble essentiel d'ouvrir un dialogue avec les enseignants francoontariens, afin de mieux comprendre les représentations qu'ils se font de leurs compétences professionnelles. En effet, plusieurs chercheurs (Gather-Thurler, 1994; Perrenoud, 1993; Sarason, 1990) insistent sur l'importance, pour le succès de toute réforme, de prendre en considération les acteurs eux-mêmes. Puisque l'Ordre des enseignantes et des enseignants de l'Ontario, en établissant les normes de la pratique enseignante, a identifié les compétences nécessaires au maintien de ces normes (Bélair, Lacasse et Lebel, 2001) à partir des référentiels de compétences déjà existants, on peut se demander jusqu'à quel point celles-ci sont porteuses de sens pour les enseignants francoontariens. Dans cette optique les référentiels de Perrenoud $(1998,2002)$ et de Gauthier (Ministère de l'Éducation du Québec, 2002) ont été retenus pour trois raisons: 1) de forts liens ont été identifiés entre ces référentiels et les Normes d'exercice de la profession enseignante élaborées par l'Ordre des enseignantes et des enseignants de l'Ontario (1996); 2) ces référentiels sont à la base des réformes éducationnelles, non seulement en Ontario, mais aussi en Suisse et au Québec; 3) le langage utilisé est issu des courants de pensée contemporains concernant les pratiques pédagogiques. 


\section{Question de recherche spécifique}

Dans le cadre de cette recherche, nous interrogeons les enseignants sur les représentations qu'ils se font de leur travail au quotidien et, par ricochet, des compétences professionnelles qu'ils croient posséder. L'analyse des rapprochements et des écarts entre ces représentations et les référentiels de Perrenoud $(1998,2002)$ et de Gauthier (MEQ, 2002) peut servir d'indicateur des changements au niveau des représentations entraînés par le Programme de perfectionnement professionnel mis de l'avant par l'Ordre des enseignantes et des enseignants de l'Ontario, tout autant que du travail qui reste encore à faire dans ce domaine.

Précisons aussi que dans le cadre de cette recherche, les représentations sont étudiées en considérant, à l'instar de Moscovici et Farr (1986), qu'une représentation est reliée à l'environnement social et que ce lien social doit toujours être interprété par rapport à un objet ou une situation donnée. Dans cette même perspective, Jodelet (1984) soutient que « la notion de représentation concerne la façon dont nous, sujets sociaux, appréhendons les événements de la vie courante, les données de notre environnement, les informations qui y circulent, etc. » (p. 360-361). Propre à un groupe, la représentation est intégrée dans le système de valeurs en fonction de l'histoire du sujet ou du groupe, de même que des contextes sociaux et idéologiques propres à leur vécu. C'est pourquoi cette étude s'intéresse à dégager les représentations sociales des enseignants qui vivent une expérience « professionnalisante ».

\section{Méthodologie}

Cette recherche s'inscrit dans une tradition de recherche qualitative interprétative (Lessard-Hébert, 1996), d'une part, pour son aspect descriptif et inductif et, d'autre part, en ce qu'elle vise à comprendre la dynamique du phénomène étudié, tel que construite par les acteurs de la situation visée (Karsenti et Savoie-Zajc, 2000). Le choix des participants, tout autant que l'élaboration des méthodes de collecte et d'analyse des données, répondent à cette orientation.

\section{Participants}

Les participants ont été recrutés parmi les enseignants de la première cohorte qui devait suivre des cours en français à l'Université d'Ottawa, en réponse aux exigences du Programme de perfectionnement professionnel, à l'été 2002. Afin d'obtenir un échantillonnage utile, une approche suggérée par Cresswell (1998) et Huberman et Miles (1994), les critères de sélection visaient à s'assurer que tous les participants à l'étude commençaient le processus de recertification exigé par l'Ordre des enseignantes et des enseignants de l'Ontario et connaissaient les programmes d'études prescrits par le gouvernement Harris. Les participants devaient donc avoir au moins deux ans d'expérience en enseignement. De plus, l'échantillon devait inclure des enseignants des niveaux primaire et secondaire. Dix-neuf personnes se sont portées volontaires pour cette recherche. Huit personnes ont été retenues et six d'entre elles se sont présentées au groupe de discussion. Ces dernières avaient en moyenne 13 ans d'expérience en enseignement. Au moment des entretiens individuels, deux candidats se sont retirés. Les entretiens individuels ont donc été menés avec quatre participants ayant en moyenne 10 ans d'expérience. 


\section{Procédures de collecte et d'analyse des données}

La collecte des données s'est faite en deux temps, soit un groupe de discussion, mené en juillet 2002, suivi d'un entretien individuel. Ce choix méthodologique visait à explorer en profondeur la question de recherche. Au cours du groupe de discussion, les participants ont identifié les compétences à développer afin d'être professionnel, ce qui a servi à préparer les entretiens individuels au cours desquels les participants ont pu continuer la construction de leurs représentations des compétences.

Les questions présentées au groupe de discussion avaient pour but de faire parler des compétences en enseignement et d'en évaluer l'importance. La formulation des questions est demeurée à un niveau général, pour ne pas influencer les participants et mieux saisir l'essentiel de leurs représentations, tout en maintenant la discussion proche de la pratique. Les questions suivantes ont été posées :

1) Lorsqu'on parle du domaine de l'enseignement, qu'est-ce que le mot compétence signifie pour vous?

2) Quelles compétences exercez-vous dans votre pratique?

3) Quelle est la compétence la plus importante à votre avis?

La discussion s'est terminée en demandant aux participants de définir le terme « compétence ».

En ce qui a trait à l'analyse des données du groupe de discussion, le texte intégral des propos des participants a d'abord été relu et chaque réplique numérotée. Les énoncés les plus importants ont été surlignés et les mots clés inscrits en marge. En suivant le fil de la conversation, des catégories ont émergé (compétence-définition et compétence-devenir), puis des classes (formation, savoir, savoir être et savoir faire) et des sous-classes (adaptabilité, acquis, connaissances, construction de compétences), ce qui a permis de bâtir un système de codification à l'aide du logiciel File Maker Pro 5.5. Les liens entre les catégories, les classes et les sous-classes ont ensuite été analysés et un profil des représentations des compétences professionnelles gravitant autour de quatre grands thèmes, soit les acquis expérientiels, l'adaptabilité, les connaissances et la formation, a été constitué pour chaque participant.

À la suite du groupe de discussion, la chercheure s'est inspirée de la méthode d'analyse de construits personnels (Chevalier, 2005; Kelly, 1955) afin de structurer les entretiens individuels. II s'agissait de faire parler chaque participant de ses propres représentations des compétences professionnelles, sans préjudice par rapport à ses croyances sur le sujet. Dans cette optique, chaque participant devait d'abord réorganiser, sur un tableau d'affichage, les mots clés associés aux compétences identifiées lors du groupe de discussion. Les mots clés jugés non pertinents pouvaient être placés dans une enveloppe de recyclage. Deuxièmement, le participant devait examiner son propre profil d'un enseignant compétent et indiquer s'il était d'accord ou non avec l'organisation proposée. Des fiches avaient été mises à la disposition des participants s'il s'avérait nécessaire d'ajouter des mots clés au tableau. Troisièmement, tenant compte de ce qu'il venait d'assembler au tableau, le participant devait formuler, oralement ou par écrit, une définition du concept de « compétence ». Enfin, le participant devait établir des liens entre les mots clés réorganisés sur le tableau d'affichage et les référentiels de Perrenoud $(1998,2002)$ et de Gauthier (MEQ, 2002).

Les données des entretiens individuels ont été analysées à partir du même système de codification que celui du groupe de discussion. Les catégories, les classes et les sous-classes ont été modifiées pour permettre au logiciel 
d'établir des liens entre les profils de compétences des participants et les référentiels de compétences des chercheurs choisis.

\section{Présentation des résultats}

Les résultats sont présentés en deux parties. La première porte sur l'analyse des propos que les participants ont entretenus suite aux questions posées lors du groupe de discussion. La seconde s'intéresse aux discours suscités pendant les différentes étapes des entretiens individuels.

\section{Groupe de discussion}

La première tâche confiée au groupe de discussion portait sur les représentations générales des compétences professionnelles en enseignement. À cette étape, il ne s'agissait pas de forcer une définition précise du terme « compétence » mais plutôt de favoriser la collaboration entre les participants et laisser la réflexion mener vers une réponse ouverte. Lors de cette discussion, les participants ont fait un remue-méninges tout en donnant des exemples directement tirés de leur pratique en enseignement, ce qui a permis de répondre, du même coup, à la deuxième question « Quelles compétences exercez-vous dans votre pratique? »

Au cours de cette étape, les compétences en lien avec les trois savoirs classiques, soit « savoir, savoir faire et savoir être », issus de la pédagogie du pionnier Pestalozzi (Soëtard, 1994), sont automatiquement ressortis, sans qu'aucune intervention n'ait été faite pour susciter l'établissement de liens entre les savoirs. Les extraits suivants permettent de saisir la valeur centrale de ces trois savoirs dans les discours des enseignants :

\section{Savoir}

- ...la première chose, c'est une maîtrise absolue de sa didactique. II faut connaître son contenu, il faut savoir de quoi on va parler. Le comment vient après mais pour moi, la plus grande compétence à avoir c'est de connaître sa matière.

- ...plus que la didactique, la maîtrise de la langue est importante.

\section{Savoir être}

- .... il y a une partie de ces compétences que je pense qui viennent de un, avec l'expérience, mais aussi avec le côtoiement avec des personnes d'expérience.

- ...tant que tu te connaîtras pas dans une situation donnée, c'est souvent ça qui va faire la différence entre le succès ou l'échec...

- Ça retourne à la question d'adaptabilité tant vis-à-vis du contenu qu'au groupe auquel on fait affaire. Et puis l'adaptabilité ça va assez loin, on peut greffer des valeurs sociales à ça. Comment expliquer à de petits êtres humains comment interagir, qu'est-ce qu'on va leur prodiguer comme contenu, etc.

\section{Savoir faire}

- De plus en plus, on a des gros groupes difficiles à enseigner. Moi j'enseigne au secondaire, je pense à des problèmes de comportements, je pense à des nouveaux enseignants qui entrent en classe et c'est assez difficile. 
La lecture de ces extraits laisse entrevoir que la grande majorité des compétences identifiées sont d'ordre général et s'appliquent à tous les contextes. Cependant, certaines, dont la maîtrise de la langue, révèlent une préoccupation particulière au contexte minoritaire.

Après avoir fait le tour des compétences relatives à la pratique de l'enseignement, les participants ont été invités à organiser celles-ci selon une hiérarchie d'importance. Les réponses indiquent qu'il n'y a pas de consensus chez les participants quant à la classification des compétences en terme d'importance. Devant cette divergence de points de vue, les mots clés ressortis de la discussion entourant cette question ont été mis en parallèle avec les paradigmes de l'enseignant professionnel identifiés dans le référentiel de Paquay (1994: voir Paquay et Wagner, 2001) et les trois savoirs classiques issus de la pédagogie de Pestalozzi. Ceci permet de voir que la structure sousjacente qui émerge naturellement du discours des participants touche à toutes les caractéristiques essentielles d'un enseignant compétent identifiées par ces auteurs.

\section{Tableau 1}

\section{Parallèle entre les mots clés ressortis du groupe de discussion, le référentiel Paquay et les savoirs classiques de Pestalozzi}

\begin{tabular}{|c|c|c|}
\hline Référentiel de Paquay & Mots clés ressortis du groupe de discussion & Savoirs classiques \\
\hline $\begin{array}{l}\text { - Un « maître instruit », qui maîtrise } \\
\text { des savoirs }\end{array}$ & $\begin{array}{l}\text { Maîtrise } \\
\text { - les connaissances } \\
\text { - la didactique } \\
\text { - la langue } \\
\text { Connaît } \\
\text { - ce qu'est l'enseignement } \\
\text { - sa matière } \\
\text { - la langue }\end{array}$ & Savoir \\
\hline $\begin{array}{l}\text { - Un « technicien », qui a acquis } \\
\text { systématiquement des savoir-faire }\end{array}$ & $\begin{array}{l}\text { Comprend } \\
\text { - les attentes } \\
\text { - les exigences de la job } \\
\text { Possède } \\
\text { - les qualifications nécessaires } \\
\text { - l'habileté d'évaluer } \\
\text { - des compétences mécaniques et des } \\
\text { compétences organiques } \\
\text { - des outils en poche pour la gestion de } \\
\text { classe }\end{array}$ & Savoir faire \\
\hline $\begin{array}{l}\text { - Un « praticien artisan » qui a } \\
\text { acquis des schémas d'action } \\
\text { contextualisés }\end{array}$ & $\begin{array}{l}\text { Sait transmettre } \\
\text { - des connaissances } \\
\text { - des habiletés } \\
\text { - de plusieurs façons } \\
\text { - des valeurs sociales } \\
\text { S'adapte } \\
\text { - aux exigences de multiples tâches } \\
\text { - au contexte } \\
\text { - au groupe-classe }\end{array}$ & Savoir faire \\
\hline $\begin{array}{l}\text { - Un « praticien réflexif » qui s'est } \\
\text { construit un « savoir d'expérience » } \\
\text { systématique et communicable, plus } \\
\text { ou moins théorisé }\end{array}$ & $\begin{array}{l}\text { A } \\
\text { - une approche qui est centrée sur } \\
\text { l'élève } \\
\text { - une approche qui prend compte des } \\
\text { styles }\end{array}$ & Savoir faire \\
\hline
\end{tabular}




\section{Tableau 1 (suite)}

\section{Parallèle entre les mots clés ressortis du groupe de discussion, le référentiel Paquay et les savoirs classiques de Pestalozzi}

\begin{tabular}{|c|c|c|}
\hline Référentiel de Paquay & Mots clés ressortis du groupe de discussion & Savoirs classiques \\
\hline $\begin{array}{l}\text { - Un « acteur social » engagé dans } \\
\text { des projets collectifs et conscient } \\
\text { des enjeux anthropo-sociaux des } \\
\text { pratiques quotidiennes }\end{array}$ & $\begin{array}{l}\text { Est capable } \\
\text { - d'apprendre de ses collègues } \\
\text { - de gérer le changement } \\
\text { - de gérer les crises } \\
\text { - de cultiver la collaboration } \\
\text { - de réfléchir }\end{array}$ & Savoir être \\
\hline $\begin{array}{l}\text { - Une « personne » en relation et en } \\
\text { développement de soi }\end{array}$ & $\begin{array}{ll}\text { Est } & \\
& \text { - confiant en soi (et se connaît) } \\
\text { - pro-actif } \\
\text { - agréable (a de l'entregent) } \\
\text { - présent (dégage de l'énergie, a le sens } \\
\text { d'émerveillement) } \\
\text { - enthousiaste } \\
\text { - passionné }\end{array}$ & Savoir être \\
\hline
\end{tabular}

Pour terminer la discussion de groupe, les participants devaient tenter de définir le mot « compétence ». ॥ était entendu qu'à ce stade de l'étude, il s'agissait d'une première tentative de formalisation de la pensée des participants. Quoique approximatives, ces définitions suggèrent que l'image mentale que les participants se faisaient du mot compétence lors du groupe de discussion, correspond à ce qu'un expert est capable d'accomplir dans l'action. À titre d'illustration, voici les définitions de trois participants qui ont tenté une formulation plus précise du concept de compétence :

- Une compétence pour moi, c'est d'avoir en possession toutes les habiletés nécessaires pour accomplir le travail demandé (...) au niveau de l'enseignement, parce qu'on est des touche-àtout, qu'on parle de la gestion de classe, de la connaissance de soi, de la connaissance des styles d'apprentissage, connaissance des styles d'enseignement, mettons-le large.

- ...est-ce qu'on cherche une compétence mécanique, comme un médecin, sa compétence sera de couper avec son bistouri. En enseignement c'est tellement organique, c'est tellement large que chercher des compétences, me semble qu'on peut tricoter ben longtemps avant d'arriver...

- Moi je dis qu'on est tous les ogues: psychologue, sociologue, tous les eurs : médiateurs, organisateurs.

\section{Entretiens individuels}

À partir des résultats de l'analyse du groupe de discussion, les entretiens ont été structurés rappelons-le, en quatre temps. Ce découpage devait permettre d'arriver à un consensus avec le participant quant aux résultats du groupe de discussion, d'une part, et de comprendre le sens que le participant accordait aux compétences des référentiels de Perrenoud $(1998,2002)$ et de Gauthier (MEQ, 2002), d'autre part.

Au cours de la première étape, chaque participant a réorganisé les mots clés émanant du groupe de discussion en utilisant une stratégie différente pour exprimer sa pensée. Cet exercice a donc donné lieu à quatre 
représentations qui devaient servir de base pour l'interprétation des liens avec les référentiels de Perrenoud (1998, 2002) et de Gauthier (MEQ, 2002). D'une manière métaphorique, ces quatre représentations se décrivent comme suit :

1) la représentation en fenêtres s'organise autour de cases qui s'ouvrent les unes sur les autres à la manière d'un système d'exploitation informatique. Avec l'enseignant comme point de départ, les compétences les plus importantes sont ensuite placées, puis elles s'ouvrent sur les compétences considérées comme secondaires;

2) la représentation cyclique est organisée comme un système solaire avec l'apprenant au centre et les trois savoirs qui gravitent autour de lui;

3) la représentation proximo-distale part de l'enseignant, au centre, pour se diriger vers l'extérieur, c'est-àdire vers l'acte éducatif, qui demande de contrôler les savoirs et les compétences;

4) la représentation hiérarchique organise les mots clés et les compétences en ordre d'importance, comme dans une nomenclature systématique.

Pour la deuxième étape des entretiens, soit les réactions sur leur profil personnel de compétences, les participants se sont tous dits d'accord que ce profil était représentatif de leur définition d'un enseignant compétent. Certains ont apporté des précisions en lien avec le contexte particulier de la salle de classe dans laquelle ils travaillent, ainsi que sur la formation des enseignants, telles que :

- ... c'est que la formation-là, j'vas être ben franc, l'université fait certainement sa part, mais y'a rien comme être en salle de classe pour apprendre.

- ...t'essaies d'appliquer ça dans la mesure du possible, avec les jeunes que t'as, avec la façon dont tu te sens ce matin-là, puis tout ça, y'a une question là-dedans, y'a un élément externe qui vient aussi jouer, t'as beau avoir ça, mais ça va pas s'appliquer tous les jours à 100\%.

- ...la façon de transmettre tes connaissances, c'est là la différence, c'est ça qui va faire la différence chez tes élèves, si y vont se l'approprier ou non. Si y vont croire dans ce que t'enseignes. Parce qu'on fait pas juste enseigner une matière, ces enfants-là, c'est notre société de demain. C'est des êtres en devenir. Et quand tu enseignes, tu enseignes un peu de toi.

- ...faire face à des problèmes de gestion de classe, y'a pas assez de formation. Ah c'est terrible, ça c'est pourri! C't'une tape en plein visage!

La troisième étape des entretiens, soit la formulation d'une définition de compétence en enseignement, fait, encore une fois, ressortir les trois savoirs classiques (savoir, savoir être et savoir faire). Selon les participants, pour être un enseignant compétent la personne doit :

1) être agréable, enthousiaste

2) être redevable face aux parents

3) savoir donner le goût d'apprendre aux enfants

4) être capable de travailler avec l'équipe école

5) être capable de s'adapter aux situations changeantes de l'école

6) être capable de gérer les situations de crise 
7) être capable de s'adapter aux différents besoins de ses élèves

8) être capable de livrer son programme d'études le plus efficacement possible, en tenant compte des différents styles cognitifs de ses élèves, afin de les motiver à apprendre.

Ces définitions s'apparentent davantage à une définition formelle de compétence que celles ressorties lors du groupe de discussion, dans la mesure où elles possèdent toutes une composante de processus.

La quatrième étape des entretiens visait à établir des liens entre les représentations des compétences des participants et celles définies dans les référentiels de Perrenoud $(1998,2002)$ et de Gauthier (MEQ, 2002), dont Bélair, Lacasse et Lebel (2001) se sont servis pour dégager les compétences relatives aux normes adoptées par l'Ordre des enseignantes et des enseignants de l'Ontario. Les participants ont émis des commentaires sur les compétences du référentiel de Perrenoud en analysant chaque compétence individuellement, tandis que pour le référentiel de Gauthier, ils ont plutôt formulé des commentaires généraux. C'est pourquoi les paragraphes qui suivent se limitent à présenter l'interprétation que font les participants des compétences du référentiel de Perrenoud.

Pour deux de ces compétences, soit « Pratiquer une évaluation formatrice » et «Aller vers un moindre cloisonnement disciplinaire » les participants ont eu des interprétations très semblables entre eux, mais s'écartant quelque peu de celles de Perrenoud $(1998,2002)$. Alors que ce dernier insiste sur le fait que l'évaluation est formatrice au sens où elle doit être spontanée et contribuer à construire une aptitude à l'autoévaluation, les participants ont tous interprété cette compétence comme une évaluation formative dans le sens où le "maître », soucieux d'optimiser son enseignement, procède à une évaluation systématique afin de situer l'apprenant dans ses apprentissages :

- Au fil de ce que l'élève fait, je vérifie au travers.

- Oui, c'est très important pour moi aussi, là, très important qu'un élève, et puis j'aime bien leur écrire des commentaires, parce que t'as pas tout le temps la chance de verbaliser ou de les féliciter, t'sais j'veux dire.

De même, pour Perrenoud, le moindre cloisonnement disciplinaire signifie clairement que les situations ouvertes, générées par des problèmes réels que les élèves doivent résoudre, mobilisent nécessairement des éléments de plusieurs disciplines. Pour les participants, cette compétence touche leur capacité à faire de l'interdisciplinarité dans le cadre d'un enseignement de type transmissif :

- ...exactement, pis pas nécessairement avec le curriculum. Moi, là, rester dans le curriculum... Présentement, j'enseigne en $6^{e}$ puis he, si j'ai à me servir du latin, de la Grèce antique, de Rome, d'la Chine, c'que tu veux, j'vas l'faire, t'sé j'vais déborder, parce que je suis là en même temps pour aider les jeunes à se développer une culture générale.

- ....interdisciplinaire, parce que encore là, enseigner un objectif, heu une attente particulière, faut qu'elle soit rattachée à quelque chose qui est significatif et signifiant, pis c'est dans les autres disciplines que t'enseignes...

- ...comme quand ils sont tout petits, y sont en première année, t'enseignes à lire! Mais pourquoi on enseigne à lire? Y'apprennent à lire pas juste pour apprendre à lire, plus tard y lisent pour apprendre, alors faut que ce soit signifiant, alors c'est là où une situation interdisciplinaire dans l'sens que tu te sers de tes autres disciplines...

- $\quad$ Ah oui, ben ça avec la matière, ben ça, j'veux dire moi avec ma matière, c'est la base de tous les cours. J'vais aller en musique pour faire connaître les grands musiciens, on n'a pas de 
cours en musique comme ça. Qui est Chopin? Hein? Qui est, vois-tu, j'peux l'faire en français, j'peux aller en musique, les arts...

Pour quatre autres compétences, soit « Créer ou utiliser d'autres moyens d'enseignement », « Adopter une planification souple, improviser », «Établir un nouveau contrat didactique » et « Convaincre les élèves de changer de métier », les significations individuelles qu'en dégagent les participants s'écartent autant les unes des autres que de celles que leur accorde Perrenoud $(1998,2002)$. Ces quatre compétences se situent au cœur des nouvelles pédagogies et, pour cet auteur, les situations d'apprentissage doivent être authentiques et adaptées au groupe classe, ce qui exige une volonté chez l'enseignant de prendre des risques, de partir d'idées incomplètes et de les faire évoluer avec les élèves, et d'adopter une relation pédagogique plus démocratique. Les interprétations des participants, pour leur part, semblent conserver une représentation de la pédagogie dite « traditionnelle » comme l'illustrent les exemples suivants :

\section{Créer ou utiliser d'autres moyens d'enseignement}

- C'est faire des parenthèses pour stimuler l'intelligence...

- Oui, surtout à cause du TPCL1 et à cause des PEl ${ }^{2} \ldots$

- C'est de s'adapter et avoir de la flexibilité...

\section{Adopter une planification souple, improviser}

- II faut faire des liens avec le monde...

- Cela dépend de la population d'élèves...

- C'est une demi-réalité à cause du curriculum...

- C'est plus facile quand on a de l'expérience...

\section{Établir un nouveau contrat didactique}

- C'est l'enseignant qui doit avoir autorité sur les contenus, surtout en 1 e année...

- C'est bon, surtout avec les élèves du niveau appliqué...

\section{Convaincre les élèves de changer de métier}

- « convaincre » est un mot trop fort...

- Oui, c'est de l'autonomie sociale...

- Difficile à atteindre à cause du curriculum...

- Je laisse les élèves enseigner à partir de leurs projets de recherche à la fin de l'année. Moi, je prends une pause...

De la même manière qu'ils l'avaient fait au cours du groupe de discussion, et même si chaque compétence des référentiels de Perrenoud $(1998,2002)$ et de Gauthier (MEQ, 2002) présentée lors des entretiens individuels avait été placée sur une fiche contenant une explication de la compétence, les participants ont interprété ces compétences en s'appuyant sur leur pratique. Par exemple, alors que la compétence «Établir un nouveau contrat didactique » vise à modifier la relation éducative pour que l'apprenant soit impliqué activement dans ses apprentissages, les participants insistent pour que le contrôle des apprentissages reste entre les mains de l'enseignant. Pour la compétence «Convaincre les élèves de changer de métier, » alors que Perrenoud soutient que le nouveau métier d'élève doit

1 Test Provincial de Compétences Linguistiques

2 Plan d'Éducation Individualisé

Volume I, numéro 1, 2005 
permettre à l'apprenant de collaborer avec l'enseignant pour élaborer des situations-problèmes ou des projets, les discours des participants indiquent clairement que cette collaboration est encore au stade de latence.

On remarque également que le sens accordé par les participants à la définition d'un enseignant compétent diffère de celui de Perrenoud $(1998,2002)$. Entre autres, les participants mettent l'accent sur la personnalité de l'enseignant et ses qualités intrinsèques telles qu'être agréable, enthousiaste et savoir donner le goût d'apprendre aux enfants. De plus, alors que Perrenoud mise sur les pédagogies différenciées et la métacognition, les participants font référence aux approches pédagogiques axées sur la transmission de connaissances et centrées sur l'apprenant.

Signalons enfin que la formation initiale et continue, ainsi que la croissance personnelle ont été considérées comme des éléments de haute importance pour tous les participants.

\section{Pistes de réflexion}

Les résultats de cette étude montrent que les représentations que les enseignants se font de leurs compétences professionnelles sont solidement ancrées dans une longue tradition de la culture scolaire. Cette tradition fait en sorte que ces derniers interprètent le concept de compétence, de même que les compétences que les chercheurs proposent, à partir de leur connaissance de ce qu'est l'enseignement et de leurs expériences antérieures. Les écarts dans leurs représentations des compétences professionnelles par rapport aux définitions présentées dans les référentiels de Perrenoud $(1998,2002)$ et de Gauthier (MEQ) se répercutent sur les Normes d'exercice de la profession enseignante adoptées par l'Ordre des enseignantes et des enseignants de l'Ontario, puisque les liens de parenté entre ces référentiels et les normes ont été clairement établis par Bélair, Lacasse et Lebel (2001). Les enseignants ontariens se retrouvent donc dans une situation pédagogique influencée par des représentations construites au cours de réformes antérieures (Davidson, 2003) qui font maintenant partie intégrante de leur réalité quotidienne. Dans la ligne de pensée de la NGP, en autant que les élèves réussissent bien aux tests provinciaux, les enseignants sont rassurés que leur interprétation des compétences professionnelles est congruente avec celle de l'Ordre. Cette situation est susceptible de contribuer au maintien du conflit implicite entre valeurs et praxis, et de renforcer les résistances au changement.

Ces résistances ne sont pourtant pas anormales. Dans la foulée des travaux qui portent sur l'amélioration du système éducatif, il apparaît que toute réforme prend du temps à s'intégrer dans la réalité quotidienne. À la manière d'un paquebot, explique Perrenoud (2002), le système éducatif est une lourde machine dotée d'une grande force d'inertie. Ainsi, les politiques éducatives exigent plus de trois ou quatre ans pour présenter des signes intérieurs d'appropriation. À cet égard, Perrenoud avance qu'il faut un dispositif de pilotage précis et explicite si l'on désire vraiment accroître les chances de garder le cap sur le changement. Pour sa part, Bélair (2003) soutient que l'école est une institution qui répond au droit fondamental de l'éducation et, pour ce faire, elle ne doit pas se plier aux exigences du marché, mais bien à celles d'une pédagogie visant à préparer le citoyen de demain, habile et capable de s'adapter à différentes situations. Ces constatations obligent à dépolitiser l'école suffisamment pour qu'elle puisse avoir la liberté et le temps de remplir sa mission. Nos résultats indiquent effectivement que le changement des représentations de compétences professionnelles prend du temps et que les pressions politiques, aussi fortes soient-elles, ne semblent pas pouvoir accélérer le processus. 
Parallèlement à ce phénomène d'inertie, la lenteur dans le changement des représentations des enseignants peut s'expliquer en partie par les frustrations causées par les contradictions dans les discours de l'Ordre des enseignantes et des enseignants de l'Ontario. D'une part, l'Ordre emprunte ses normes de compétences aux recherches qui s'inscrivent dans un cadre socioconstructiviste contraire à la philosophie dominante du Ministère de l'éducation de l'Ontario qui, dans l'esprit de St-Germain (2001b), mise sur l'obligation des résultats, la Nouvelle Gestion Publique et l'école en tant qu'entreprise redevable au public. D'autre part, les Normes de la profession enseignante et le Programme de perfectionnement professionnel sous-entendent que les enseignants manquent de professionnalisme et que la meilleure façon de corriger cette situation est de les obliger à suivre des cours sous la menace de retirer leur certificat de compétence. Ainsi, l'organisme qui était supposé aider à autoréglementer la profession devient une menace qui pèse sur les enseignants. Bien qu'il fait référence à la situation du Québec, Tardif (2004) décrit une telle approche en affirmant : «D'un côté, il s'agit de faire peur aux enseignants en leur disant que l'ordre professionnel sera un monstre bureaucratique et disciplinaire qui va les contrôler, les châtier et les punir; de l'autre côté, on veut leur faire croire que le même ordre sera la solution à tous leurs maux, qu'lls seront enfin reconnus publiquement comme de véritables professionnels et mériteront de la sorte le respect qui leur est dû » (p. 5). II est fort probable que ces contradictions dans la mission de l'Ordre des enseignantes et des enseignants de l'Ontario nourrissent non seulement la discorde qui existe encore aujourd'hui entre l'Ordre et ses membres, mais aussi les résistances face au changement et à l'amélioration du système éducatif dans son ensemble. II convient donc de s'interroger sur la nature et les fonctions des dispositifs à mettre en œuvre pour s'assurer que non seulement les discours en matière de compétences soient congruents entre les chercheurs et l'Ordre des enseignantes et des enseignants de l'Ontario, mais aussi que l'avènement des ordres professionnels ne soit pas détracteur à l'égard du travail que les enseignants accomplissent.

Malgré les retombées de la première expérience de professionnalisation ontarienne, il demeure que la tentative du Programme de perfectionnement professionnel force à mieux comprendre la dynamique du processus de développement des compétences professionnelles des enseignants et ainsi, à réorienter les discours politiques qui s'attachent à l'excellence en enseignement. À la suite de l'expérience que l'Ontario a vécue depuis 1996, on remarque déjà un changement de direction dans ces discours. La présente recherche s'intéressait à des problèmes fort à propos par rapport au climat politique dans lequel elle a été menée. C'est particulièrement parce qu'elle a été menée avant l'entrée au pouvoir du gouvernement McGuinty, qu'elle a pu mettre en évidence les divergences entre les discours des enseignants, ceux de l'Ordre des enseignantes et des enseignants de l'Ontario et ceux des chercheurs. Avec le gouvernement McGuinty au pouvoir, le ministre de l'éducation, Gerard Kennedy, initie un projet de loi proposant de modifier la Loi de 1996 sur l'Ordre des enseignantes et des enseignants de l'Ontario et d'annuler le Programme de perfectionnement professionnel. Le ministre semble également se montrer ouvert à un dialogue avec les enseignants. Entre autres, le 13 mai 2004, il a publié une lettre ouverte aux enseignants expliquant son intention de faire valoir leur travail, afin que ces derniers puissent bénéficier de la reconnaissance sociale qui leur est due. Le ministre a aussi formé une table ronde de partenariat en éducation susceptible de faciliter un échange sur la manière de construire l'excellence en enseignement et de permettre aux élèves de réaliser leur plein potentiel.

Ces constatations semblent dès lors soulever comme hypothèse qu'en dépit des réformes successives, des restructurations, de l'empressement vers l'amélioration de la qualité de l'éducation, le changement éducatif prend du temps à s'incarner dans la réalité. La professionnalisation de l'enseignement est le résultat de plusieurs décennies de 
volonté d'amélioration de la condition enseignante. Dans ce sens, cette recherche permet de questionner non seulement l'orientation de la formation initiale et continue offerte aux enseignants, mais aussi d'initier une réflexion sur l'accompagnement des nouveaux enseignants qui font leur entrée dans la profession. Entre autres, en ayant une meilleure compréhension des contraintes reliées au changement des représentations des compétences en enseignement par les enseignants expérimentés, il est possible de mieux orienter les programmes de mentorat pour les nouveaux enseignants à partir des besoins qui se font sentir dans leur milieu de travail, plutôt que de miser exclusivement sur l'expérience du mentor en enseignement.

\section{Conclusion}

Vouloir gérer la professionnalisation des enseignants entre la peur et la promesse de reconnaissance sociale est contraire aux valeurs qui priment actuellement dans le contexte éducatif. Tous les apprenants, y compris les enseignants, doivent trouver du sens pour se construire des savoirs et réaliser des apprentissages durables. En rétrospective, la réforme initiée par le gouvernement Harris en Ontario s'est accrochée à une idéologie qui refuse de considérer les valeurs inhérentes au métier d'enseignant, ce qui a grandement influencé la manière dont l'Ordre des enseignantes et des enseignants de l'Ontario a assumé son pouvoir et imposé le Programme de perfectionnement professionnel. Les répercussions en ont été particulièrement démoralisantes pour les enseignants. En cherchant à comprendre la manière dont les enseignants se représentent leurs compétences professionnelles, cette recherche a dégagé une partie des connaissances et des valeurs que possède cette communauté de pratique, ce qui s'avère essentiel comme point de départ vers une professionnalisation authentique.

L'orientation proposée par le ministre de l'éducation actuel, Gerard Kennedy, semble favorable à une entrée en dialogue qui ne peut qu'être un pas en avant dans la construction de la profession enseignante et vers la congruence des regards sur la professionnalisation. L'idée de partenariat constitue un premier élément de contrôle par les membres de la profession, élément qui permettra de faire entendre la voix des acteurs et d'ajuster le perfectionnement professionnel en conséquence. Reste à voir dans quelle mesure l'Ordre des enseignantes et des enseignants de l'Ontario tirera profit de cet échange entre les partis pour mettre en place des dispositifs permettant de renforcer les compétences des acteurs de cette pratique sociale. La poursuite des recherches dans ce domaine devrait atteindre un plus grand nombre d'enseignants et utiliser des techniques plus approfondies d'analyse de construits personnels afin de mieux comprendre les représentations de compétences professionnelles qui guident actuellement l'acte pédagogique des enseignantes et des enseignants franco-ontariens. De plus, la recherche devrait porter une attention particulière à la compétence langagière, qui est ressortie dans le discours des participants. À I'heure actuelle, aucune des normes ou des compétences qui s'en dégagent ne porte spécifiquement sur le développement de compétences langagières, élément non négligeable du quotidien des enseignants qui oeuvrent en milieu minoritaire francophone. De telles études pourraient contribuer à la conception d'un programme de professionnalisation des enseignants davantage ajusté aux besoins des acteurs. 


\section{Remerciements}

Mes sincères remerciements aux enseignantes et enseignants, mes partenaires de recherche, qui ont accepté de participer à cette étude dans un moment où leur identité professionnelle était remise en question par les discours politiques.

\section{Références}

Association des enseignantes et des enseignants franco-ontariens (AEFO) (2003). Pourquoi l'AEFO dit NON. En bref, 494. http://franco.ca/aefo/enbref/494.htm (14 septembre 2005).

Bélair, L. (2003). Entre territoires aux frontières trop définies. In Y. de Saint-Do (dir.), Territoires éducatifs et gouvernance. Actes du colloque international : Territoires éducatifs et gouvernance, 14-16 avril 2003. Clermont-Ferrand, Presses universitaires Blaise Pascal.

Bélair, L., Lacasse, R. et Lebel, C. (2001). Les Normes d'exercice de la profession enseignante: un rationnel entourant les compétences de l'enseignant et de l'enseignante. Rapport de recherche. Toronto, Ordre des enseignantes et des enseignants de l'Ontario.

Chevalier, J. M. (2005). The Social Analysis System. Problem Domain. http://www.sas-pm.com/ (3 novembre 2005).

Creswell, J. W. (1998). Qualitative Inquiry and Research Design, Choosing Among Five Traditions. Thousand Oaks, Sage.

Davidson, A.-L. (2003). La réforme Harris et la professionnalisation de l'enseignement en Ontario : un processus controversé. In $\mathrm{H}$. Duchesne (dir.), Recherche en éducation francophone en milieu minoritaire : regards croisés sur une réalité mouvante. Winnipeg, Presses universitaires de Saint-Boniface, 147-174.

Department of Education and Skills (Grande-Bretagne) (2003). Department of Education and Skills Homepage. http://www.dfes.gov.uk (10 octobre 2004).

Department of Education, Science and Training (Australie) (2004). Department of Education, Science and Training Homepage. http://www.dest.gov.au (10 août 2004).

Develay, M. (2001). Propos sur les sciences de l'éducation. Paris, Éditions Sociales Françaises.

Gather-Thurler, M. (1994). Relations professionnelles et culture des établissements scolaires : au-delà du culte de l'individualisme ? Revue française de pédagogie, 109, 19-39.

Gérin-Lajoie, D. (2001). Les défis de l'enseignement en milieu minoritaire, le cas de l'Ontario. Le renouvellement de la profession enseignante : tendances, enjeux et défis des années 2000, Éducation et Francophonie, 29 (1). http://www.acelf.ca/c/revue/revuehtml/29-1/02-Gerin-Lajoie.html\#h-3.1 (31 mai 2005).

Gohier, C. (2002). Les finalités de l'éducation, Éducation et francophonie, 30 (1), printemps 2002. www.acelf.ca/revue/XXX1/index.html (10 octobre 2004).

Gouvernement de l'Ontario (1996). Loi de 1996 sur l'Ordre des enseignantes et des enseignants de l'Ontario, L.O. 1996, c. 12. http://www.iijcan.org/on/legis/loi/1996c.12/20041104/tout.html (14 septembre 2005).

Gouvernement de l'Ontario (2004). Communiqué: une nouvelle loi reconnaît les enseignantes et enseignants comme des professionnels. http://ogov.newswire.ca/ontario/GPOF/2004/05/13/c2897.html?Imatch=\&lang=_f.html (14 septembre 2005).

Gouvernement du Québec (2002). Avis de l'office des professions du Québec sur l'opportunité de constituer un ordre professionnel des enseignantes et des enseignants. Québec, Bibliothèque nationale du Québec. http://www.opq.gouv.qc.ca/PDF/Avisenseignants.pdf (3 novembre 2005).

Huberman, M. A. et Miles, M. B. (1991). Analyse des données qualitatives. Recueil de nouvelles méthodes. Bruxelles, De Boeck Université.

Jodelet, D. (1984). La représentation sociale : phénomènes, concepts et théories. In S. Moscovici (dir.), Psychologie Sociale. Paris, Presses universitaires de France.

Karsenti, T. et Savoie-Zajc, L. (2000). Introduction à la recherche en éducation. Sherbrooke, Éditions du CRP.

Kelly, G. A. (1955). Psychology of Personal construct. New York, Norton.

Lessard-Hébert, M., Goyette, G. et Boutin, G. (1996). La Recherche qualitative, fondements pratiques (2éd). Montréal, Éditions Nouvelles.

Ministère de l'Éducation du Québec (2002). Formation initiale. Québec, Direction de la formation et de la titularisation des enseignants. http://www.meq.gouv.qc.ca/dftps/ (10 octobre 2004).

Ministère de l'Éducation du Québec (2003). Éducation Québec. http://www.meq.gouv.qc.cal (10 octobre 2004).

Ministère de l'Éducation de l'Ontario (2002). Appuyer l'excellence dans l'enseignement, Programme ontarien d'évaluation du personnel enseignant. Toronto, Imprimeur de la reine pour l'Ontario. http://www.edu.gov.on.ca/fre/document/nr/00.05/testfsf.html (21 novembre 2002). 
Ministère de l'Éducation de l'Ontario (2004). Appuyer l'excellence dans l'enseignement. Fiche de renseignement sur le programme de perfectionnement professionnel. Toronto, Imprimeur de la reine pour l'Ontario. http://www.edu.gov.on.ca/fre/teacher/learningfs.html (1er janvier 2004).

Ministère de l'éducation nationale, de l'enseignement supérieur et de la recherche (2002). Éducation.fr. : Le portail de référence sur l'éducation. http://www.education.fr/page.php?P=data/accueil/ (10 octobre 2004).

Moscovici, S. et Farr, R. (1984). Social representations. Cambridge, Cambridge University Press.

Ontario Secondary School Teacher Federation (OSSTF) (2004). Restructuring the College of Teachers. Education Watch, 2, 2.

Ordre des enseignantes et des enseignants de l'Ontario (1996). Les normes d'exercice de la profession enseignante. Toronto, OEO. www.oct.on.ca/fr/College/Publications/standards.pdf (10 octobre 2004).

Ordre des enseignantes et des enseignants de l'Ontario (2000). Maintenir, assurer et démontrer la compétence de la profession enseignante. Réponse à la requête de la ministre de l'Éducation sur un programme d'évaluation du personnel enseignant. Pour parler profession, juin 2000. http://www.oct.ca/fr/CollegePublications/PS/juin_2000/cover.htm (10 octobre 2004).

Paquay, L. et Wagner, M.-C. (2001). Compétences professionnelles privilégiées dans les stages et en vidéo-formation. In L. Paquay, M. Altet, É. Charlier et P. Perrenoud (dir.), Former des enseignants professionnels ( $3^{\mathrm{e}}$ éd). Bruxelles, De Boeck.

Perrenoud, P. (1993). Formation initiale des maîtres et professionnalisation du métier. Revue des Sciences de l'Éducation, 19(1), 59-76.

Perrenoud, P. (1998). Construire des compétences dès l'école. Paris, Éditions Sociales Françaises.

Perrenoud, P. (2002). Dix principes pour rendre le système éducatif plus efficace. Genève, Faculté de psychologie et des sciences de l'éducation.

Routhier Boudreau, L. (2002). L'Ordre a assez joué le jeu du gouvernement. En Bref, 477. http://franco.ca/aefo/enbref/477.htm (10 septembre 2005).

Routhier Boudreau, L. (2003). Recertification : maintenons le boycottage! En Bref, 494. http://franco.ca/aefo/enbref/494.htm (10 septembre 2005).

St-Germain, M. (2001). Origines et incidences des nouveaux rapports de force dans la gestion de l'éducation. Éducation et francophonie, 29(2). http://www.acelf.ca/c/revue/revuehtml/29-2/00-Liminaire.html (14 septembre 2005).

St-Jarre, C. et Dupuy-Walker, L. (dir.) (2001). Le temps en éducation, regards multiples. Sainte-Foy, Presses de l'Université du Québec.

Sarason, S. (1990). The Predictable Failure of Educational Reform. San Francisco, Jossey-Bass.

Soëtard, M. (1994). Johann Heinrich Pestalozzi, 1746-1827. Perspectives : revue trimestrielle d'éducation comparée, 24(1-2), 307320.

Tardif, M. (2004). La profession enseignante au Québec : entre Charybde et Scylla? Formation et Profession, août 2004, 5-7.

Tardif, M. et Gauthier, C. (1999). Pour ou contre un ordre professionnel des enseignantes et des enseignants au Québec? Québec, Presses de l'Université Laval.

UNESCO (2001). Données mondiales de l'éducation (4e éd.), Cédérom. Genève, Bureau international d'éducation. 